\title{
EPOS Model and Ultra High Energy Cosmic Rays
}

\author{
T. Pierog ${ }^{\mathrm{a}}$ and K. Werner ${ }^{\mathrm{b}}$ \\ ${ }^{\text {a}}$ Forschungszentrum Karlsruhe, Institut für Kernphysik, \\ Postfach 3640, Karlsruhe, Germany

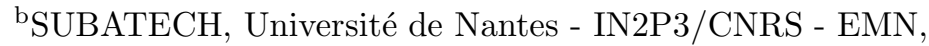 \\ Nantes, France
}

Interpretation of extensive air showers (EAS) experiments results is strongly based on air shower simulations. The latter being based on hadronic interaction models, any new model can help for the understanding of the nature of cosmic rays. The EPOS model reproducing all major results of existing accelerator data (including detailed data of RHIC experiments) has been introduced in air shower simulation programs CORSIKA and CONEX few years ago. The new EPOS 1.99 has recently been updated taking into account the problem seen in EAS development using EPOS 1.61. We will show in details the relationship between some EPOS hadronic properties and EAS development, as well as the consequences on the model and finally on cosmic ray analysis.

\section{INTRODUCTION}

Air shower simulations are a very powerful tool to interpret ground based cosmic ray experiments. However, most simulations are still based on hadronic interaction models being more than 10 years old. Much has been learned since, in particular due to new data available from the SPS and RHIC accelerators.

In this paper, we discuss air shower simulations based on EPOS, the latter one being a hadronic interaction model, which does very well compared to RHIC data [1, and also other particle physic experiments (especially SPS experiments at CERN). But used in air shower simulation program like CORSIKA [2] or CONEX [3], some results where in contradiction with KASCADE data 4, while it was better for other experiments [5].

Due to the constrains of particle physics, air shower simulations using EPOS present a larger number of muons at ground [6. On the other hand, we will explain in this paper, how the contrains given by cosmic ray experiments can compensate the lack of accelerator data in some given kinematic regions (very forward) to improve hadronic interaction models and in particular the new EPOS 1.99.

\section{EPOS Model}

One may consider the simple parton model to be the basis of high energy hadron-hadron interaction models, which can be seen as an exchange of a "parton ladder" between the two hadrons. In EPOS, the term "parton ladder" is actually

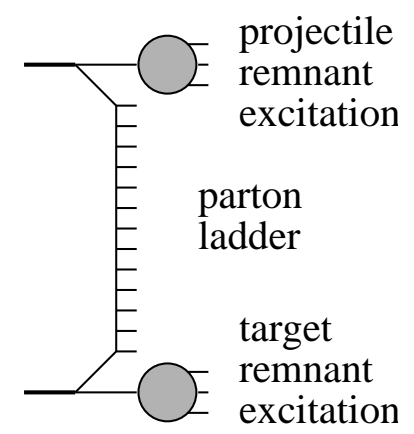

Figure 1. Elementary parton-parton scattering: the hard scattering in the middle is preceded by parton emissions attached to remnants. The remnants are an important source of particle production even at RHIC energies.

meant to contain two parts [7: the hard one, as discussed above, and a soft one, which is a purely phenomenological object, parameterized in Regge pole fashion. 
In additions to the parton ladder, there is another source of particle production: the two off-shell remnants, see fig. 1] We showed in ref. [8] that this "three object picture" can solve the "multi-strange baryon problem" of conventional high energy models, see ref. [9].

Hence EPOS is a consistent quantum mechanical multiple scattering approach based on partons and strings [7, where cross sections and the particle production are calculated consistently, taking into account energy conservation in both cases (unlike other models where energy conservation is not considered for cross section calculations [10]). Nuclear effects related to Cronin transverse momentum broadening, parton saturation, and screening have been introduced into EPOS [11. Furthermore, high density effects leading to collective behavior in heavy ion collisions are also taken into account [12].

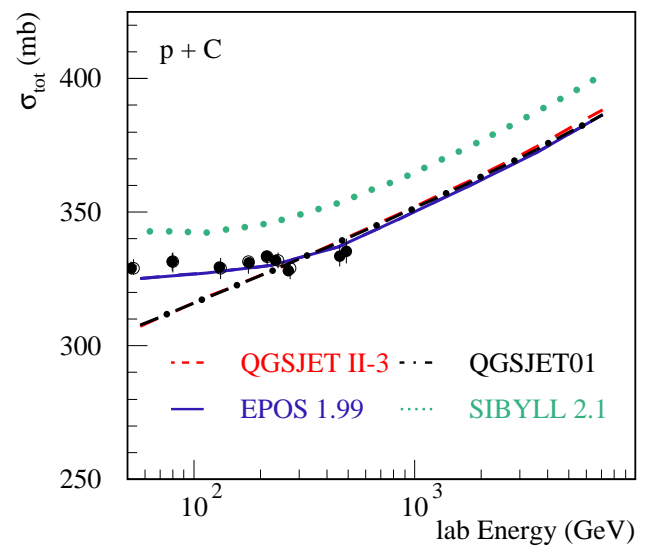

Figure 2. Total cross section of proton-carbon interactions. EPOS 1.99, QGSJETII, QGSJET01 and SIBYLL 2.1 hadronic interaction models (lines) are compared to data [13 (points)

Energy momentum sharing and remnant treatment are the key points of the model concerning air shower simulations because they directly influence the multiplicity and the inelasticity of the model. At very high energies or high densities, the so-called non-linear effects described in [11] are particularly important for the extrapolation for EAS and it's one of the parts which has been changed in EPOS 1.99.

\subsection{Cross section and inelasticity}

We learned from KASCADE data [4, that the energy carried by hadrons in EPOS 1.61 simulations is too low. It means than the showers are too old when they reach ground and it was due to a problem in the calculation of the nuclear cross section and to a too large remnant break-up at high energy (leading to a high inelasticity).

To improve the predictive power of the model, the effective treatment of non-linear effects describe in 11 has been made consistent to describe both proton-proton, hadron-nucleus and nucleusnucleus data with a unique saturation scale which can be fixed thanks to proton-proton cross section and Cronin effect in dAu collisions at RHIC. Details will be published in a dedicated article.

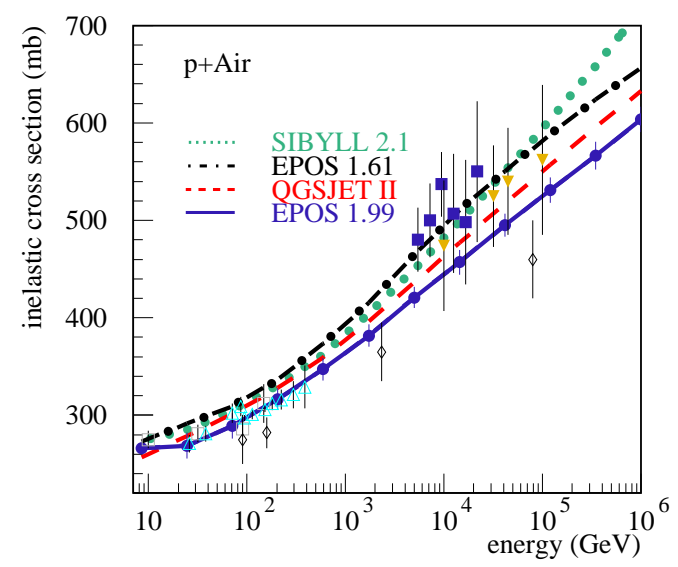

Figure 3. Inelastic cross section of proton-air interactions. EPOS 1.99, QGSJETII, EPOS 1.61 and SIBYLL 2.1 hadronic interaction models (lines) are compared to data of air shower experiment (points).

The EPOS 1.99 (full line) proton-carbon total cross section is shown Fig 2, It is now in very good agreement with the data 13 and with the other hadronic interaction model used for air shower physics QGSJET01 [14] (dashdotted line), QGSJETII [15] (dashed line) and SIBYLL [16] (dotted line). In fig [3, the extrapolation to proton-air data up to the highest energies is shown in comparison with measurement from cosmic ray experiments. The error bar for EPOS 1.99 represents the uncertainty 
due to the definition of the inelastic cross section as measured by cosmic ray experiment: 1 . In comparison with EPOS 1.61 (dash-dotted line), the EPOS 1.99 cross section has been notably reduced.

\subsection{Particle production}

Thanks to a Monte Carlo, first the collision configuration is determined: i.e. the number of each type of Pomerons exchanged between the projectile and target is fixed and the initial energy is shared between the Pomerons and the two remnants. Then particle production is accounted from two kinds of sources, remnant decay and cut Pomeron. A Pomeron may be regarded as a twolayer (soft) parton ladder attached to projectile and target remnants through its two legs. Each leg is a color singlet, of type qव, qqq or qqq from the sea, and then each cut Pomeron is regarded as two strings, cf. Fig. 诂. a)

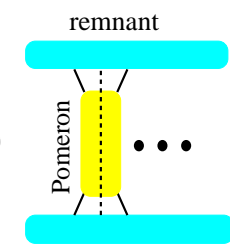

b)

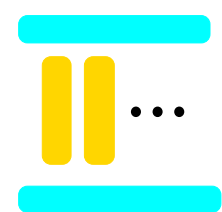

remnant

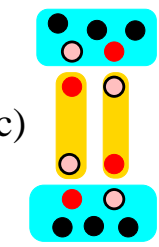

d)

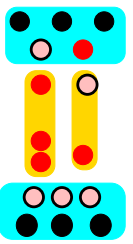

e)

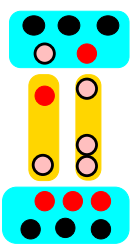

Figure 4. a) Each cut Pomeron is regarded as two strings b). c) The most simple and frequent collision configuration has two remnants and only one cut Pomeron represented by two $q-\bar{q}$ strings. d) One of the $\bar{q}$ string ends can be replaced by a qq string end. e) With the same probability, one of the q string ends can be replaced by a $\overline{\text { qq }}$ string end.

\footnotetext{
${ }^{1}$ The difference between the top and the bottom of the error bars is the part of the cross-section where secondary particles are produced without changing the projectile (target diffraction). Cross section of other models includes this target diffraction (top of error bars).
}

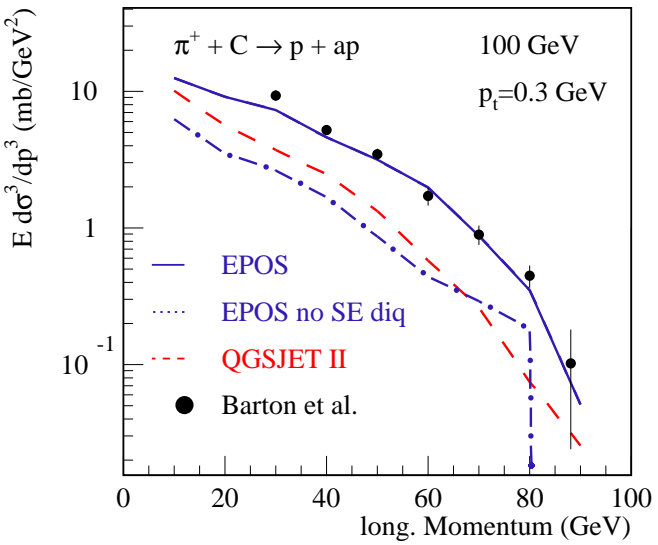

Figure 5. Model comparison: longitudinal momentum distributions of pion carbon collisions at $100 \mathrm{GeV}$ from EPOS with (full) or without (dash-dotted) sting-end diquarks and QGSJETII (dashed) compared to data [17.

It is a natural idea to take quarks and antiquarks from the sea as string ends for soft Pomeron in EPOS, because an arbitary number of Pomerons may be involved. In addition to this soft Pomerons, hard and semihard Pomerons are treated differently.

Thus, besides the three valence quarks, each remnant has additionally quarks and antiquarks to compensate the flavours of the string ends, as shown in fig. 4t. According to its number of quarks and antiquarks, to the phase space, and to an excitation probability, a remnant decays into mesons, (anti)baryons [8]. Furthermore, this process leads to a baryon stopping phenomenon in which the baryon number can be transfered from the remnant to the string ends (for instance in $4 \mathrm{~d}$, depending on the process, the $3 \bar{q}+3 q$ can be seen as 3 mesons or a baryon-antibaryon pair).

In case of meson projectile, this kind of diquark pair production at the string ends leads to an increase of the (anti)baryon production in the forward production in agreement with low energy pion-nucleus data [17] as shown fig. [5. As a consequence it is part of the larger number of muons in EAS simulations with EPOS.

Compared to EPOS 1.61, EPOS 1.99 has a reduced excitation probability at high energy, in- 
creasing the number of protons in the forward direction and reducing the inelasticity.

\section{AIR SHOWERS}

In the following, we discuss air shower simulations, based on the shower programs CONEX, using the old EPOS 1.61 (dash-dotted line), the new EPOS 1.99 (full line) and QGSJETII(dashed line) (as a reference) as high energy hadronic interaction model in KASCADE experiment energy range.

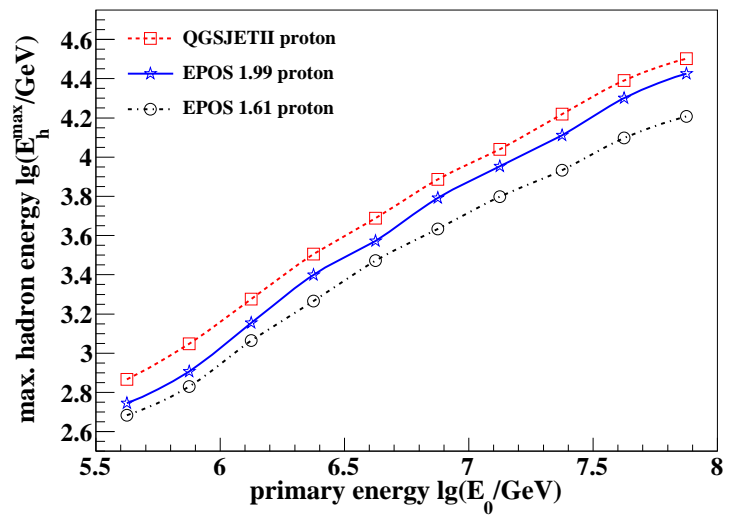

Figure 6. Maximum hadron energy as a function of the primary energy for proton induced showers using EPOS 1.99 (full line), EPOS 1.61 (dashdotted line) and QGSJETII (dashed line) as high energy hadronic interaction model.

The effect of the reduced cross section and inelasticity is clearly visible on the maximum energy of hadrons at ground as shown fig. 6. The shower being younger at ground with EPOS 1.99, the maximum energy is up to $60 \%$ higher than in the previous release 1.61. The results are now close to QGSJETII results but with a different slope due to a different elongation rate. As a consequence, EPOS 1.99 does not have the problems pointed out in [4 anymore and should be compatible with KASCADE data.

\section{Summary}

EPOS is a new interaction model constructed on a solid theoretical basis. It has been tested very carefully against all existing hadronic data, also those usually not considered important for cosmic rays. In EAS simulations, EPOS provides more muons than other models, which was found to be linked to an increased diquark production in both string ends and string fragmentation. The new EPOS 1.99 has a reduced cross section and inelasticity compared to the previous EPOS 1.61 which leads to deeper shower development. This would solve the problem with KASCADE data. But since the number of muons and the elongation rate are different than in the other models, the resulting analysis will be significantly different.

\section{REFERENCES}

1. R. Bellwied. Acta Phys. Hung., A27:201-204, 2006.

2. D. Heck et al. FZKA-6019, 1998.

3. T. Bergmann et al. Astropart. Phys., 26:420432, 2007.

4. W. D. Apel et al., J. Phys. G: Nucl. Part. Phys. 36:035201, 2009

5. A. V. Glushkov et al., JETP Lett., 87:190 2008.

6. T. Pierog and K. Werner. Phys. Rev. Lett. 101:171101 2008.

7. H. J. Drescher et al. Phys. Rept., 350:93-289, 2001.

8. F. M. Liu et al. Phys. Rev., D67:034011, 2003.

9. M. Bleicher et al. Phys. Rev. Lett., 88:202501, 2002.

10. M. Hladik et al. Phys. Rev. Lett., 86:35063509, 2001.

11. K. Werner et al. Phys. Rev., C74:044902, 2006.

12. K. Werner. Phys. Rev. Lett., 98:152301, 2007.

13. U. Dersch et al. Nucl. Phys. B579:277, 2000.

14. N. N. Kalmykov, S. S. Ostapchenko, and A. I. Pavlov, Nucl. Phys. Proc. Suppl. 52B (1997) 17-28.

15. S. Ostapchenko. Phys. Rev., D74:014026, 2006.

16. R. Engel, T. K. Gaisser, P. Lipari, and T. Stanev, in Proceedings of 26th ICRC (Salt Lake City) vol. 1, p. 415, 1999.

17. D. S. Barton et al. Phys. Rev., D27:2580, 1983. 